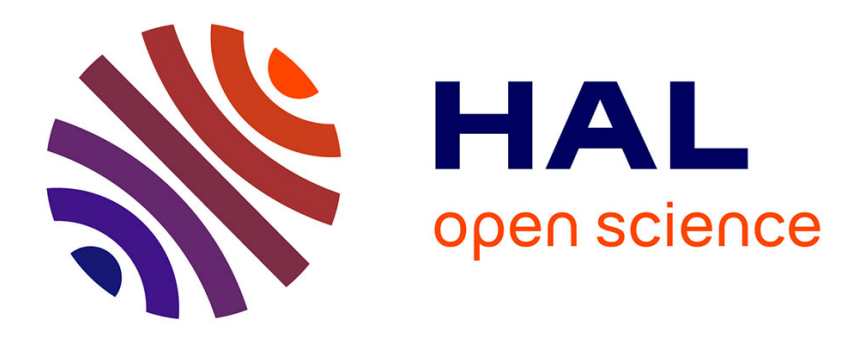

\title{
In vitro selection of fluoroquinolone resistance in Brucella melitensis
}

\author{
N. Ravanel, B. Gestin, M. Maurin
}

\section{To cite this version:}

N. Ravanel, B. Gestin, M. Maurin. In vitro selection of fluoroquinolone resistance in Brucella melitensis. International Journal of Antimicrobial Agents, 2009, 34 (1), pp.76. 10.1016/j.ijantimicag.2009.01.002 . hal-00556326

\section{HAL Id: hal-00556326 https://hal.science/hal-00556326}

Submitted on 16 Jan 2011

HAL is a multi-disciplinary open access archive for the deposit and dissemination of scientific research documents, whether they are published or not. The documents may come from teaching and research institutions in France or abroad, or from public or private research centers.
L'archive ouverte pluridisciplinaire HAL, est destinée au dépôt et à la diffusion de documents scientifiques de niveau recherche, publiés ou non, émanant des établissements d'enseignement et de recherche français ou étrangers, des laboratoires publics ou privés. 


\section{Accepted Manuscript}

Title: In vitro selection of fluoroquinolone resistance in Brucella melitensis

Authors: N. Ravanel, B. Gestin, M. Maurin

PII: $\quad$ S0924-8579(09)00025-9

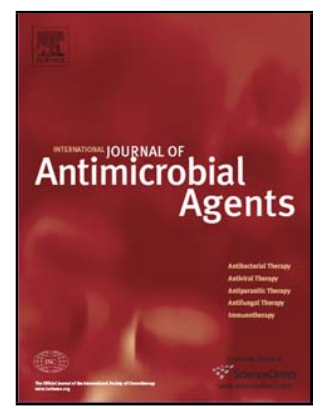

DOI: doi:10.1016/j.ijantimicag.2009.01.002

Reference: ANTAGE 2964

To appear in: International Journal of Antimicrobial Agents

Received date: 7-11-2008

Revised date: $31-12-2008$

Accepted date: 4-1-2009

Please cite this article as: Ravanel N, Gestin B, Maurin M, In vitro selection of fluoroquinolone resistance in Brucella melitensis, International Journal of Antimicrobial Agents (2008), doi:10.1016/j.ijantimicag.2009.01.002

This is a PDF file of an unedited manuscript that has been accepted for publication. As a service to our customers we are providing this early version of the manuscript. The manuscript will undergo copyediting, typesetting, and review of the resulting proof before it is published in its final form. Please note that during the production process errors may be discovered which could affect the content, and all legal disclaimers that apply to the journal pertain. 


\title{
In vitro selection of fluoroquinolone resistance in Brucella melitensis
}

\begin{abstract}
N. Ravanel, B. Gestin, M. Maurin *
Laboratoire de Bactériologie, Centre Hospitalier Universitaire de Grenoble, Université Joseph Fourier, BP217, 38043 Grenoble cedex 9, France Laboratoire Adaptation et Pathogénie des Microorganismes, CNRS UMR 5163, Université Joseph Fourier, Grenoble, France
\end{abstract}

Received 7 November 2008; accepted 4 January 2009

Keywords: Brucella; Brucellosis; Resistance mechanisms; Fluoroquinolones; DNA gyrase; Efflux

* Corresponding author. Tel.: +334 767654 79; fax: +33 476765912.

E-mail address: mmaurin@chu-grenoble.fr (M. Maurin). 


\section{Abstract}

Moxifloxacin-resistant mutants of Brucella melitensis 16M [moxifloxacin minimum inhibitory concentration $(\mathrm{MIC})=1 \mathrm{mg} / \mathrm{L}]$ were selected in order to characterise fluoroquinolone resistance mechanisms in this species. Eight independent mutants were obtained, with moxifloxacin MICs of $16-32 \mathrm{mg} / \mathrm{L}$. The mutants displayed variable cross-resistance levels to other fluoroquinolone compounds, but no increased resistance to aminoglycosides, tetracycline, rifampicin, macrolides or cotrimoxazole. Sequencing of type II topoisomerase-encoding genes ( $g y r A, g y r B$, parC and parE), which are natural targets for fluoroquinolones, revealed a gyrA mutation leading to the amino acid substitution Ala83Val (Escherichia coli numbering system) in five mutants with a moxifloxacin MIC of $32 \mathrm{mg} / \mathrm{L}$, whereas no mutation was found in the remaining three mutants with an MIC of $16 \mathrm{mg} / \mathrm{L}$. Phenylalanine-arginine- $\beta$ naphthylamide dihydrochloride, an efflux pump inhibitor, reduced moxifloxacin MICs by a factor of two to eight in all resistant mutants. In B. melitensis, fluoroquinolone resistance may arise from gyrA mutation and efflux pump overexpression mechanisms. 


\section{Introduction}

Brucella spp. are aetiological agents of brucellosis, a worldwide zoonosis [1]. Terrestrial and marine mammals are natural reservoirs for these bacteria. Human contamination usually occurs via direct contact with infected domestic animals or via the digestive route after consumption of contaminated raw milk or milk products. Pasteurisation of milk and eradication of brucellosis in cattle has dramatically reduced the incidence of human brucellosis in developed countries. However, brucellosis remains endemic and a public health problem in many countries where these prophylactic measures have not been implemented, especially around the Mediterranean Sea, in the Middle East, in central Asia and in some parts of Africa and South America [2].

Two antibiotic combinations, i.e. doxycycline + rifampicin or doxycycline plus an aminoglycoside (e.g. streptomycin or gentamicin), remain the mainstay in brucellosis therapy [1]. Therapy alternatives may be needed in patients with severe adverse effects as well as in children $<8$ years old and in pregnant women in whom tetracyclines are contraindicated [1]. On the other hand, relapse rates of $10-15 \%$ are still observed with these antibiotic combinations despite prolonged therapy. Combinations of trimethoprim/sulphamethoxazole (co-trimoxazole) or a macrolide with either rifampicin or an aminoglycoside have been used for decades, but with higher relapse rates [1]. The fluoroquinolones have recently been considered as a promising alternative in the treatment of human brucellosis $[3,4]$. These antibiotics display low minimum inhibitory concentrations (MICs) against Brucella spp. in vitro [5-10] and have good oral bioavailability and a large volume of distribution including in eukaryotic cells where Brucella sp. multiply $[11,12]$. They are usually well tolerated 
although, as for tetracyclines, they are contraindicated in young children and pregnant women. However, the use of fluoroquinolone monotherapy in brucellosis patients has led to frequent relapses and failures [13-15], and the combination of a fluoroquinolone with another antibiotic (especially rifampicin) is no more effective than currently available antibiotic regimens $[3,4,16]$. Thus, fluoroquinolones are currently not recommended as a first-line drug to treat brucellosis patients [3].

Several hypotheses have been raised to explain the discrepancies between the in vitro and in vivo activities of fluoroquinolones. First, fluoroquinolones have no bactericidal activity against Brucella spp. either in vitro $[17,18]$ or in animal models $[19,20]$. Moreover, Brucella sp. multiply in acidic phagosomes both in professional and non-professional phagocytic cells [11,12]. Although fluoroquinolones are concentrated within eukaryotic cells, their activity is dramatically reduced at acidic $\mathrm{pH}$ $[17,18]$. Finally, in vivo selection of fluoroquinolone-resistant mutants in Brucella sp. has been evoked [21]. In vitro, only low variations in fluoroquinolone MICs between various Brucella strains has been reported $[7-10,18]$, although there are rare exceptions [22].

Two major fluoroquinolones resistance mechanisms have been described so far [23,24]: (i) mutations in type II topoisomerase genes, encoding DNA gyrase and type IV topoisomerase, which are the natural targets of fluoroquinolone action; and (ii) overexpression of efflux pumps reducing drug accumulation in bacteria. Turkmani et al. [25] recently reported selection of gyrA-mediated fluoroquinolone resistance in Brucella abortus. In the present study, we further explored fluoroquinolone resistance mechanisms in Brucella melitensis. 


\section{Material and methods}

\subsection{Bacterial strains}

Brucella melitensis 16M (ATCC 23456) was used in all experiments. Brucella melitensis $16 \mathrm{M}$ was grown on Columbia agar supplemented with $5 \%$ sheep blood (bioMérieux, Lyon, France) at $37^{\circ} \mathrm{C}$ in a $5 \% \mathrm{CO}_{2}$-enriched atmosphere in a biosafety level 3 laboratory. Escherichia coli ATCC 25922 and Staphylococcus aureus ATCC 25923 were used as control strains for MIC determination.

\subsection{Antibiotics}

The following antibiotics were tested: moxifloxacin (Bayer Pharma, Puteaux, France), ciprofloxacin (Bayer Pharma), levofloxacin (sanofi-aventis, Paris, France), doxycycline (Arrow, Lyon, France), rifampicin (sanofi-aventis), gentamicin (Dakota Pharma, Paris, France), co-trimoxazole (Roche, Neuilly-sur-Seine, France) and erythromycin (CSP, Cournon, France). Antibiotics were dissolved as recommended by the manufacturers, prepared in aqueous solutions at a concentration of $2 \mathrm{mg} / \mathrm{mL}$ and stored at $-80^{\circ} \mathrm{C}$ until use.

\subsection{Selection of fluoroquinolone-resistant mutants}

Brucella melitensis $16 \mathrm{M}$ was grown on Columbia agar with $5 \%$ sheep blood at $37^{\circ} \mathrm{C}$ in $5 \% \mathrm{CO}_{2}$ atmosphere for 2 days. A bacterial inoculum was prepared in MuellerHinton broth (MHB) (AES Laboratory, Combourg, France) at 0.5 McFarland standard. Then, $20 \mu \mathrm{L}$ of this suspension was added to each well of a 96-well microtitre plate. 
MHB (180 $\mu \mathrm{L} /$ well) containing two-fold serial dilutions of moxifloxacin $(0.06-128 \mathrm{mg} / \mathrm{L}$ of final concentrations) was added to each row of the microtitre plate. Thus, the experimental design allowed eight independent mutants to be selected. The microplate was incubated at $37^{\circ} \mathrm{C}$ in a $5 \% \mathrm{CO}_{2}$ atmosphere. After $3-5$ days incubation for the first 17 days of the experiment, or after 7-10 days incubation for the remaining 51 days of the experiment, the plate was visually examined for bacterial growth and moxifloxacin susceptibility values were noted. For each row, bacterial growth observed in the well containing the highest moxifloxacin concentration was harvested and dispensed (10 $\mu \mathrm{L} /$ well) in a new microtitre plate containing MHB (190 $\mu \mathrm{L} /$ well) with two-fold serial dilutions of moxifloxacin as above. Twelve subcultures were performed for a total period of 68 days, allowing progressive selection of eight moxifloxacin-resistant mutants. These mutants were then passaged eight times on antibiotic-free blood agar plates to test the stability of the resistant phenotypes and individual colonies were selected for further analysis. During the antibiotic resistance selection process, the intermediate-resistant populations were also harvested and kept frozen at $-80^{\circ} \mathrm{C}$ for further analysis.

\subsection{Minimum inhibitory concentration determination}

For $B$. melitensis $16 \mathrm{M}$ and isogenic resistant mutants, MICs were determined using a microdilution technique. For each strain, a $0.5 \mathrm{McF}$ arland bacterial suspension was prepared in MHB and dispensed (180 $\mu \mathrm{L} /$ well) in 96-well microtitre plates. Antibiotics were added (20 $\mu \mathrm{L} /$ well $)$ at ten times the desired final concentrations. Plates were incubated at $37^{\circ} \mathrm{C}$ in a $5 \% \mathrm{CO}_{2}$ atmosphere and MICs were read after 3 days incubation. MICs were defined as the lowest antibiotic concentration that completely 
inhibited visual growth. For E. coli ATCC 25922 and S. aureus ATCC 25923, MICs were determined using the same procedure but the incubation time was $18 \mathrm{~h}$.

\subsection{DNA gyrase and topoisomerase IV gene sequencing}

Bacterial suspensions were prepared by harvesting a single colony from each mutant in $1 \mathrm{~mL}$ of sterile distilled water. Bacteria were inactivated by heating at $90^{\circ} \mathrm{C}$ for $2 \mathrm{~h}$. DNA was extracted using the QIAmp DNA Mini Kit (QIAGEN S.A., Courtaboeuf, France) according to manufacturer's instructions. Primers used for polymerase chain reaction (PCR) amplification and DNA sequencing are described in Table 1 and were purchased from MGW-Biotech (Roissy, France). PCR primers were first designed to amplify the quinolone resistance-determining regions (QRDRs) of gyrA, gyrB, parC and parE using the B. melitensis $16 \mathrm{M}$ genome sequence available in GenBank (NC 003317 and NC 003318). For B. melitensis $16 \mathrm{M}$ and two fluoroquinolone-resistant mutants, whole DNA sequences of gyrA and parC genes were determined using the primer pairs described in Table 1. The specificity of the primers was checked using BLAST alignment (BLASTn; National Center for Biotechnology Information, Bethesda, MD). PCR was performed using the Pwo Master Kit (Roche Diagnostics, Meylan, France) according to the manufacturer's instructions. Amplifications were carried out in $50 \mu \mathrm{L}$ volumes containing $0.4 \mu \mathrm{M}$ reverse and forward primers and $5 \mu \mathrm{L}$ of DNA template. PCR was initiated by denaturation at $94{ }^{\circ} \mathrm{C}$ for $5 \mathrm{~min}$, followed by 35 cycles at $94{ }^{\circ} \mathrm{C}$ for $30 \mathrm{~s}, 55^{\circ} \mathrm{C}$ for $30 \mathrm{~s}$ and $72{ }^{\circ} \mathrm{C}$ for $30 \mathrm{~s}$, and a final elongation step at $72{ }^{\circ} \mathrm{C}$ for $5 \mathrm{~min}$. The efficacy of PCR amplification was verified by agarose gel electrophoresis and ethidium bromide staining of PCR products. PCR products were then purified using Sephadex ${ }^{\mathrm{TM}}$-containing Millipore MAHV N45 plates (Sigma 
Aldrich, Saint-Quentin-Fallavier, France). DNA sequencing was performed on a CEQ2000XL apparatus using GenomeLab ${ }^{\text {TM }}$ DTCS Quick Start Kit (Beckman Coulter, Roissy, France) and a CEQ2000 Dye Terminator Cycle Sequencing Protocol (Beckman Coulter). Sequence analysis was performed using the CEQ2000XL DNA analysis system (Beckman Coulter).

\subsection{Evaluation of efflux pump overexpression}

Moxifloxacin MICs of $B$. melitensis and the eight resistant mutants were determined in the presence or absence of efflux pump inhibitors (EPIs) (Sigma-Aldrich), including verapamil $(25 \mu \mathrm{M})$, reserpine $(32.8 \mu \mathrm{M})$, carbonyl cyanide-3-chlorophenylhydrazone (CCCP) (25-100 $\mu \mathrm{M})$, sodium orthovanadate $(50 \mu \mathrm{M})$, phenylalanine-arginine- $\beta$ naphthylamide $(\mathrm{PA} \beta \mathrm{N})(38.5 \mu \mathrm{M})$ and 1-naphthylmethyl-piperazine $(88.4 \mu \mathrm{M})$. The activity of each EPI was also tested against $B$. melitensis $16 \mathrm{M}$. Since PA $\beta N$ was the only EPI that significantly reduced moxifloxacin MICs in resistant mutants, its activity was further tested at concentrations ranging from $38.5 \mu \mathrm{M}$ to $616.5 \mu \mathrm{M}$ on moxifloxacin, levofloxacin and ciprofloxacin MICs to determine a dose-effect relationship. All experiments with EPIs were performed three times to verify consistency.

\subsection{NorMI and NorMII gene sequencing}

Norml and Normll are currently the only efflux pumps that have been characterised as functionally active in $B$. melitensis $16 \mathrm{M}$ [26]. They belong to the MATE (multidrug and toxic extrusion) efflux pump family and have been shown to be specifically susceptible to PA $\beta N$ [26]. Whole NorMI and NorMII genes plus a 250 bp upstream 
DNA fragment were amplified and sequenced in $B$. melitensis $16 \mathrm{M}$ and two fluoroquinolone-resistant mutants using the primers indicated in Table 1 to check for the presence of mutations in the genes or in promoters.

\section{Results}

\subsection{Selection of fluoroquinolone resistance in Brucella melitensis $16 \mathrm{M}$}

Following a total of 12 passages (i.e. 68 days) of $B$. melitensis $16 \mathrm{M}$ in the presence of moxifloxacin concentrations ranging from $0.25 \mathrm{mg} / \mathrm{L}$ to $256 \mathrm{mg} / \mathrm{L}$, eight independent resistant mutants were selected, termed M1 to M8 (Table 2). First, five passages were performed every 3-5 days with no evident resistance selection. Then, seven additional passages were performed every $7-10$ days and resistance selection rapidly occurred. Moxifloxacin resistance was stable in all mutants after eight further subcultures in antibiotic-free medium.

\subsection{Minimum inhibitory concentration determination}

In MHB, B. melitensis $16 \mathrm{M}$ displayed MICs of $0.125 \mathrm{mg} / \mathrm{L}$ for doxycycline, $0.5 \mathrm{mg} / \mathrm{L}$ for rifampicin, $0.5 \mathrm{mg} / \mathrm{L}$ for gentamicin, $1 \mathrm{mg} / \mathrm{L}$ for levofloxacin, ciprofloxacin and moxifloxacin, and $1.6 \mathrm{mg} / \mathrm{L}$ and $8 \mathrm{mg} / \mathrm{L}$ for trimethoprim and sulphamethoxazole, respectively. MICs of the same antibiotics for S. aureus ATCC 25923 and E. coli ATCC 25922 were in the expected ranges.

Among the eight $B$. melitensis mutant strains, five displayed an MIC to moxifloxacin of $32 \mathrm{mg} / \mathrm{L}$, whereas the remaining three displayed an MIC of $16 \mathrm{mg} / \mathrm{L}$ (Table 2). Variable levels of cross-resistance to the other fluoroquinolones tested were found 
for the selected resistant mutants, with MICs ranging from $0.5 \mathrm{mg} / \mathrm{L}$ to $8 \mathrm{mg} / \mathrm{L}$ for ciprofloxacin and from $1 \mathrm{mg} / \mathrm{L}$ to $8 \mathrm{mg} / \mathrm{L}$ for levofloxacin (Table 2). Interestingly, ciprofloxacin and levofloxacin MICs remained unchanged for M7 despite a 16-fold increase in the moxifloxacin MIC. MICs to doxycycline, rifampicin, gentamicin and cotrimoxazole were unchanged in the eight moxifloxacin-resistant mutants compared with the B. melitensis $16 \mathrm{M}$ wild-type strain.

\subsection{Determination of DNA gyrase and topoisomerase IV sequences}

A single mutation in the gyrA QRDR was identified in the five mutants with the highest moxifloxacin MIC (32 mg/L) in codon 67 (GCT $\rightarrow$ GTT) leading to amino acid substitution Ala67Val (Table 2). Codon 67 in B. melitensis GyrA corresponds to codon 83 in the E. coli GyrA numbering system. No mutation was found in the gyrA QRDR of the remaining three mutants with moxifloxacin MICs of $16 \mathrm{mg} / \mathrm{L}$. A 6 base insertion (i.e. CGCCAG) was found in the parE gene of mutant 8. This insertion led to two amino acid insertions, an alanine and a serine, at positions 515 and 516 in the corresponding protein. No mutations were found in gyrB or parC QRDR sequences. Determination of the whole gyrA sequence (2709 bp) in B. melitensis $16 \mathrm{M}$ and in M1 and M2 mutants (moxifloxacin MICs $16 \mathrm{mg} / \mathrm{L}$ and $32 \mathrm{mg} / \mathrm{L}$, respectively) confirmed the presence of a single mutation at position 67 in $\mathrm{M} 2$ and the absence of any gyrA mutation in M1 compared with the B. melitensis $16 \mathrm{M}$ gyrA sequence. No mutation was found in the whole sequence of parC (2328 bp) in M1 and M2 mutants compared with that of B. melitensis $16 \mathrm{M}$.

The intermediate-resistant populations were also checked for the presence of mutations in DNA gyrase- and topoisomerase-encoding genes. It was observed that 
an Ala67Val substitution in GyrA occurred in the five mutants at different steps during the selection process (Table 2). The presence of Ala67Val substitution was correlated with a higher level of cross-resistance to levofloxacin and ciprofloxacin.

\subsection{Evaluation of efflux pump overexpression}

None of the tested EPIs displayed a bacteriostatic effect against $B$. melitensis $16 \mathrm{M}$ on its own, except PA $\beta \mathrm{N}$ at concentrations $>154 \mu \mathrm{M}$, and CCCP that completely inhibited bacterial growth at concentrations ranging from $25 \mu \mathrm{M}$ to $100 \mu \mathrm{M}$. CCCP activity could not be evaluated in resistant mutants. As shown in Table 3, only PA $\beta N$ significantly reduced the moxifloxacin MIC (by a factor of two to eight) in all moxifloxacin-resistant mutants, but not in B. melitensis 16M. Furthermore, a doseeffect relationship was demonstrated (Table 3 ). PA $\beta N$ only displayed a weak effect on ciprofloxacin and levofloxacin MICs in moxifloxacin-resistant mutants at a concentration of $154 \beta \mathrm{M}$ (Table 3).

\subsection{NorMI and NorMIl gene sequencing}

No mutations were found in NorMI and NorMII genes or in the $250 \mathrm{bp}$ upstream of the genes for $B$. melitensis $16 \mathrm{M}$ and moxifloxacin-resistant mutants $\mathrm{M} 2$ and M6 (moxifloxacin MICs of 32 and $16 \mathrm{mg} / \mathrm{L}$, respectively).

\section{Discussion}

The aim of the present study was to evaluate potential resistance mechanisms to fluoroquinolones in Brucella spp. A B. melitensis strain was studied because this 
species is responsible for most human brucellosis cases in the world [1] and as potential fluoroquinolone resistance mechanisms have never been studied in this species. In vitro selection for fluoroquinolone resistance in $B$. melitensis $16 \mathrm{M}$ was easily achieved using moxifloxacin as the selecting drug, with a 16 - to 32 -fold increase in MICs in eight independent mutants. In particular, a small numbers of passages in antibiotic-containing medium was necessary to select resistant mutants when the incubation time between two passages was increased to 7-10 days. Brucella melitensis is a slow-growing bacterium. Moreover, moxifloxacin belongs to a new generation of fluoroquinolones with improved in vitro activity supposedly because of the presence of a methoxy group in $\mathrm{C} 8$. The combination of both characteristics may explain the need for prolonged incubation of Brucella cultures to select moxifloxacin-resistant mutants. Turkmani et al. [25] reported a similar observation when selecting resistance to ofloxacin and ciprofloxacin in B. abortus.

Mutations in topoisomerase-encoding genes are the leading cause of fluoroquinolone resistance in bacteria $[23,24]$. In Gram-negative bacteria, DNA gyrase is usually more susceptible to inhibition by fluoroquinolones than topoisomerase IV and thus resistance mutations occur first in the gyrA gene encoding subunit A of DNA gyrase (GyrA) $[23,24]$. These mutations usually lead to amino acid substitution in the protein sequence of the DNA-binding domain of GyrA, referred to as the QRDR, especially at hotspot positions 83 and 87 (E. coli numbering system). Frequent amino acid substitutions responsible for acquired resistance to fluoroquinolones in $E$. coli include Ser83Leu, Ser83Trp, Asp87Asn, Asp87Gly and Asp87Tyr [30]. These amino acid substitutions in the GyrA QRDR have been associated in E. coli with reduced affinity of fluoroquinolones with the enzyme-DNA complex [30]. Turkmani et al. [25] recently 
reported three types of gyrA mutations in $B$. abortus leading to fluoroquinolone resistance. These mutations occurred in different mutants and led to the following amino acid substitutions: Asp91Asn or Asp91Tyr (corresponding to substitution in codon position 87 of E. coli GyrA); and Ala87Val (corresponding to substitution in codon position 83 of $E$. coli GyrA). In the five B. melitensis $16 \mathrm{M}$ mutants with an MIC of $32 \mathrm{mg} / \mathrm{L}$ to moxifloxacin, an amino acid substitution was found at codon position 67 (Ala67Val) corresponding to position 83 in the E. coli GyrA numbering system. Sequencing of the entire gyrA and parC genes in mutant M2 did not reveal any further mutation. Thus, it is highly probable that the observed gyrA QRDR mutation explains at least partially the acquisition of moxifloxacin resistance in these five mutants. Interestingly, a correlation between MICs to fluoroquinolones and sequence variation in the gyrA QRDR, especially at codon position 83, can be established. As shown in Table 2, a serine is found at GyrA codon position 83 in bacterial species with very low MICs to ciprofloxacin and ofloxacin, e.g. wild-type strains of E. coli [31] and Mycobacterium fortuitum [28]. Ser83Ala substitution has been reported in E. coli strains with a four-fold increase in MICs to these compounds [27]. An alanine residue at codon position 83 is found naturally in Mycobacterium tuberculosis [28], B. abortus [25] and B. melitensis, which display intermediate-level resistance to ciprofloxacin and ofloxacin. Finally, Ala83Val substitution has been reported in M. tuberculosis [29], B. abortus [25] and now in B. melitensis, leading to high-level resistance to fluoroquinolones. In both M. tuberculosis and B. melitensis, Ala83Val substitution induced four- to eight-fold increases in ciprofloxacin and ofloxacin MICs. The $6 \mathrm{bp}$ insertion in parE of mutant M8 led to insertion of an alanine and a serine residue at codon positions 515 and 516 in the corresponding protein. This mutation is situated 
outside the parE QRDR and it has not been previously associated with fluoroquinolone resistance in Gram-negative bacteria.

In the three resistant mutants M1, M6 and M7, with moxifloxacin MICs of $16 \mathrm{mg} / \mathrm{L}$, no mutation was found in the QRDR of topoisomerases-encoding genes. Further sequencing of the entire gyrA and parC genes in mutant M1 did not reveal any mutation compared with the wild-type B. melitensis 16M DNA sequences. Moreover, in the five mutant lineages with Ala67Val substitution in GyrA, the substitution occurred in intermediate populations at moxifloxacin concentrations varying from 8 $\mathrm{mg} / \mathrm{L}$ to $32 \mathrm{mg} / \mathrm{L}$ according to the mutant lineage considered (Table 2 ). These observations suggested that another fluoroquinolone resistance mechanism was involved and that it preceded Ala83Val substitution. Halling and Jensen [32] have previously shown that efflux systems play an important role in resistance to macrolides in Brucella spp. We investigated the possibility of efflux pump overexpression in moxifloxacin-resistant mutants by testing MICs to this antibiotic in the presence or absence of EPIs. Only PA $\beta \mathrm{N}$ significantly reduced moxifloxacin MICs in all resistant mutants with a dose-effect relationship, but not in B. melitensis $16 \mathrm{M}$. $P A \beta N$ is known to inhibit efflux pumps of the MATE family in different Gram-negative bacterial species [33]. It is a dipeptide amide that acts as an EPI by competing with the fluoroquinolone compounds, thus precluding extrusion of these antibiotics. MATE family efflux pumps have been described in Vibrio parahaemolyticus, Pseudomonas aeruginosa, Campylobacter spp. and Bacteroides thetaiotaomicron [33]. Two putative MATE family efflux pumps have been recently described in B. melitensis, i.e. NorMI and NorMII [26]. Only NorMI has been fully characterised [26]. Thus, we can hypothesise that NorMI and/or NorMII are overexpressed in our B. melitensis 
moxifloxacin-resistant mutants. Sequencing NorMI and NorMII genes in two mutants did not reveal any mutation. However, overexpression of efflux pumps is usually related to mutation in local or global regulator genes and less frequently in structural genes [33]. The former genes are currently uncharacterised in B. melitensis.

The moxifloxacin-resistant mutants bearing GyrA A67V substitution displayed only moderate levels of cross-resistance to other fluoroquinolone compounds such as levofloxacin and ciprofloxacin. Cross-resistance can be explained by a strong structural relationship between fluoroquinolone compounds. However, as previously reported $[23,24]$, topoisomerase target preference may change according to the chemical structure of the quinolone compound considered. Also, PA $\beta N$ had only a weak effect on ofloxacin and ciprofloxacin MICs in the eight mutants, suggesting the involved efflux pumps was more effective in extruding moxifloxacin. No crossresistance was found for doxycycline, rifampicin, gentamicin and co-trimoxazole, which are first-line drugs in the antibiotic treatment of brucellosis. Although the major resistance mechanisms in bacteria to these antibiotics are different from those involved in fluoroquinolone resistance, their activity could have been altered by overexpression of efflux pump systems.

Altogether our results suggest that, as for most other Gram-negative bacteria [23,24], B. melitensis may become resistant to fluoroquinolone compounds by overexpression of efflux pumps and/or by mutations in the gyrA QRDR. The limitation of our study is that fluoroquinolone resistance mechanisms only in in vitro-selected mutant strains were characterised. Thus, the clinical relevance of our results awaits confirmation that fluoroquinolone resistance is truly occurring in clinical strains of $B$. 
melitensis, in brucellosis patients receiving fluoroquinolone therapy, and that the involved resistance mechanisms are the same in clinical strains and in in vitroselected mutant strains.

\section{Conclusion}

Our results show that moxifloxacin resistance in $B$. melitensis $16 \mathrm{M}$ first occurred by overexpression of efflux pumps. Higher resistance was then selected by mutation in the gyrA QRDR, leading to Ala67Val substitution in the GyrA QRDR. This substitution was responsible for cross-resistance to other fluoroquinolone compounds. Further work is needed to characterise better the B. melitensis efflux pumps involved in fluoroquinolone resistance, but the MATE family efflux pumps NorMI and NorMII are good candidates.

It should be emphasised that the alanine residue found at position 67 (83 in the $E$. coli numbering system) in wild-type B. melitensis GyrA is responsible for low-level resistance to fluoroquinolones, and that bacteria with low-level resistance to fluoroquinolones usually become fully resistant to these compounds through only a one-step mutation $[25,29]$. This strengthens current recommendations that fluoroquinolones should not be used as a first-line therapy in brucellosis patients [3].

Acknowledgment: The authors thank Linda Northrup for English correction.

Funding: This work was supported by a public health service grant from the Institut National de Veille Sanitaire, Saint-Maurice, France, and Direction de la Recherche Clinique, Centre Hospitalier Universitaire de Grenoble, France. 
Competing interests: None declared.

Ethical approval: Not required. 


\section{References}

[1] Pappas G, Akritidis N, Bosilkovski M, Tsianos E. Brucellosis. N Engl J Med $2005 ; 352: 2325-36$.

[2] Pappas G, Papadimitriou P, Akritidis N, Christou L, Tsianos E. The new global map of human brucellosis. Lancet Infect Dis 2006;6:91-9.

[3] Falagas ME, Bliziotis IA. Quinolones for treatment of human brucellosis: critical review of the evidence from microbiological and clinical studies. Antimicrob Agents Chemother 2006;50:22-33.

[4] Pappas G, Christou L, Akritidis N, Tsianos EV. Quinolones for brucellosis: treating old diseases with new drugs. Clin Microbiol Infect 2006;12:823-5.

[5] Bosch J, Linares J, Lopez de Goicoechea MJ, Ariza J, Cisnal MC, Martin R. Invitro activity of ciprofloxacin, ceftriaxone and five other antimicrobial agents against 95 strains of Brucella melitensis. J Antimicrob Chemother 1986;17:459_ 61.

[6] Gobernado M, Canton E, Santos M. In vitro activity of ciprofloxacin against Brucella melitensis. Eur J Clin Microbiol 1984;3:371.

[7] Khan MY, Dizon M, Kiel FK. Comparative in vitro activities of ofloxacin, difloxacin, ciprofloxacin, and other selected antimicrobial agents against Brucella melitensis. Antimicrob Agents Chemother 1989;33:1409-10.

[8] Qadri SM, Akhtar M, Ueno Y, al-Sibai MB. Susceptibility of Brucella melitensis to fluoroquinolones. Drugs Exp Clin Res 1989;15:483-5.

[9] Rubinstein E, Lang R, Shasha B, Hagar B, Diamanstein L, Joseph G, et al. In vitro susceptibility of Brucella melitensis to antibiotics. Antimicrob Agents Chemother 1991;35:1925-7. 
[10] Trujillano-Martin I, Garcia-Sanchez E, Martinez IM, Fresnadillo MJ, GarciaSanchez JE, Garcia-Rodriguez JA. In vitro activities of six new fluoroquinolones against Brucella melitensis. Antimicrob Agents Chemother 1999;43:194-5.

[11] Pizarro-Cerda J, Moreno E, Sanguedolce V, Mege JL, Gorvel JP. Virulent Brucella abortus prevents lysosome fusion and is distributed within autophagosome-like compartments. Infect Immun 1998;66:2387-92.

[12] Porte F, Naroeni A, Ouahrani-Bettache S, Liautard JP. Role of Brucella suis lipopolysaccharide $\mathrm{O}$ antigen in phagosomal genesis and in inhibition of phagosome-lysosome fusion in murine macrophages. Infect Immun 2003;71:1481-90.

[13] Lang R, Raz R, Sacks T, Shapiro M. Failure of prolonged treatment with ciprofloxacin in acute infections due to Brucella melitensis. J Antimicrob Chemother 1990;26:841-6.

[14] Al-Sibai MB, Halim MA, el-Shaker MM, Khan BA, Qadri SM. Efficacy of ciprofloxacin for treatment of Brucella melitensis infections. Antimicrob Agents Chemother 1992;36:150-2.

[15] Doganay M, Aygen B. Use of ciprofloxacin in the treatment of brucellosis. Eur J Clin Microbiol Infect Dis 1992;11:74-5.

[16] Akova M, Uzun O, Akalin HE, Hayran M, Unal S, Gur D. Quinolones in treatment of human brucellosis: comparative trial of ofloxacin-rifampin versus doxycycline-rifampin. Antimicrob Agents Chemother 1993;37:1831-4.

[17] Akova M, Gur D, Livermore DM, Kocagoz T, Akalin HE. In vitro activities of antibiotics alone and in combination against Brucella melitensis at neutral and acidic pHs. Antimicrob Agents Chemother 1999;43:1298-300. 
[18] Garcia-Rodriguez JA, Garcia Sanchez JE, Trujillano I. Lack of effective bactericidal activity of new quinolones against Brucella spp. Antimicrob Agents Chemother 1991;35:756-9.

[19] Shasha B, Lang R, Rubinstein E. Therapy of experimental murine brucellosis with streptomycin, co-trimoxazole, ciprofloxacin, ofloxacin, pefloxacin, doxycycline, and rifampin. Antimicrob Agents Chemother 1992;36:973-6.

[20] Lang R, Shasha B, Rubinstein E. Therapy of experimental murine brucellosis with streptomycin alone and in combination with ciprofloxacin, doxycycline, and rifampin. Antimicrob Agents Chemother 1993;37:2333-6.

[21] Al-Sibai MB, Qadri SM. Development of ciprofloxacin resistance in Brucella melitensis. J Antimicrob Chemother 1990;25:302-3.

[22] Kocagoz S, Akova M, Altun B, Gur D, Hascelik G. In vitro activities of new quinolones against Brucella melitensis isolated in a tertiary-care hospital in Turkey. Clin Microbiol Infect 2002;8:240-2.

[23] Hawkey PM. Mechanisms of quinolone action and microbial response. J Antimicrob Chemother 2003;51(Suppl 1):29-35.

[24] Jacoby GA. Mechanisms of resistance to quinolones. Clin Infect Dis 2005;41(Suppl 2):S120-6.

[25] Turkmani A, Psaroulaki A, Christidou A, Chochlakis D, Tabaa D, Tselentis Y. In vitro-selected resistance to fluoroquinolones in two Brucella strains associated with mutational changes in gyrA. Int J Antimicrob Agents 2008;32:227-32.

[26] Braibant M, Guilloteau L, Zygmunt MS. Functional characterization of Brucella melitensis NorMl, and efflux pump belonging to the multidrug and toxic compound extrusion family. Antimicrob Agents Chemother 2002;46:3050-3. 
[27] Tavio MD, Vila J, Ruiz J, Martin-Sanchez AM, de Anta MTJ. Mechanisms involved in the development of resistance to fluoroquinolones in Escherichia coli isolates. J Antimicrob Chemother 1999;44:735-42.

[28] Guillemin I, Jarlier V, Cambau E. Correlation between quinolone susceptibility patterns and sequences in the A and B subunits of DNA gyrase in mycobacteria. Antimicrob Agents Chemother 1998;42:2084-8.

[29] Aubry A, Veziris N, Cambau E, Truffot-Pernot C, Jarlier V, Fisher LM. Novel gyrase mutations in quinolone-resistant and -hypersusceptible clinical isolates of Mycobacterium tuberculosis: functional analysis of mutant enzymes. Antimicrob Agents Chemother 2006;50:104-12.

[30] Chen FJ, Lo HJ. Molecular mechanisms of fluoroquinolone resistance. J Microbiol Immunol Infect 2003;36:1-9.

[31] Waters B, Davies J. Amino acid variation in the GyrA subunit of bacteria potentially associated with natural resistance to fluoroquinolone antibiotics. Antimicrob Agents Chemother 1997;41:2766-9.

[32] Halling SM, Jensen AE. Intrinsic and selected resistance to antibiotics binding the ribosome: analyses of Brucella 23S rrn, L4, L22, EF-Tu1, EF-Tu2, efflux and phylogenetic implications. BMC Microbiol 2006;6:84-90.

[33] Piddock LJV. Clinically relevant chromosomally encoded multidrug resistance efflux pumps in bacteria. Clin Microbiol Rev 2006;19:382-402. 
Table 1

Oligonucleotide primers used for polymerase chain reaction (PCR) amplification and DNA sequencing of genes encoding Brucella melitensis 16M topoisomerases and efflux pumps NorMI and NorMII

\begin{tabular}{lll}
\hline Gene & Forward primer (5'-3' DNA sequence) & $\begin{array}{l}\text { Reverse primer (5'-3' DNA } \\
\text { sequence) }\end{array}$ \\
\hline gyrA QRDR & (Fw) ACGGCCTGAAGCCTGTGCAT & (Rv) CGACAGGAGGGATTCCGTGA \\
gyrA whole gene & (Fw1) CCATGAGCGTGATCGTGAG & (Rv1) CAGTTCGATGGCCGGATTG \\
& (Fw2) TTCAGGCGGTATTGCGGTC & (Rv2) CGCCTTGTTGAGCAGGAAC \\
& (Fw3) TGCAGACCTCGTTTGGCTG & (Rv3) CGCGAACGGCAATCATCTC \\
& (Fw4) CGATGAAGTGGCCGATGAG & (Rv4) CGTTGCGATTGACCTGCAC \\
& (Fw5) TTCGCCGCAACAAGCTGTC & (Rv5) GTAGCCGAATTCGCTGACC \\
& (Fw6) ATGCAGAAGCGGAGCTGTC & (Rv6) CCCCATTGCCGTTTTCCT \\
parC QRDR & (Fw3) TCGGCTCTTGAGGAACGTTA & (Rv3) \\
& & CGATGGGTTCTTCGTCTTCC \\
parC whole gene & (Fw1) GGAAAAAGTCTGATTCCGCC & (Rv1) \\
& & ATATTGGTGGCCATACCGAC \\
& (Fw2) ATGAGGAAGACGAAGAACCC & (Rv2) TCAGCTTGAACAGCGATTC \\
& (Fw3) GCTGCTTGACGATATTCGTG & (Rv3) \\
& & TGAGGTCATCGAACTCCTTG \\
\hline
\end{tabular}




\begin{tabular}{|c|c|c|}
\hline & (Fw4) CGTGCTTTTGAACTGACTG & (Rv4) \\
\hline & & AAGGCCGTCAGTATATCCTG \\
\hline & (Fw5) CGACCGACAAGCTTTTGTTC & (Rv5) ACTCGACCAGTTCTTCCTTG \\
\hline gyrB QRDR & (Fw) GTTGTCGAGAAGGTCATTCAGG & (Rv) GCGTTGAAGCCGTGCGTTTC \\
\hline parE QRDR & (Fw4) & (Rv4) \\
\hline & AGGTGCGGAATTGTTCATCGTGG & CCAAGCCCCTTGAAACGTCC \\
\hline NorMI whole & (Fw1) GACGGAACATTTGACGCGG & (Rv1) GCCCGCAAGTGCATTGAAG \\
\hline & (Fw2) CGTCCTGCGGTCTTTCTTC & (Rv2) GACCCGTGGCATGAGAAC \\
\hline & (Fw3) CCATTGCTTATGCGGGCTG & (Rv3) GCCAGCGTATCGTCATCAG \\
\hline NorMII whole & (Fw1) CCGTTCGATGTCACGCATCG & (Rv1) \\
\hline gene & & GGATCGATATATAGGTCGCC \\
\hline & (FW2) CCACCTTCATGCATCCGACAC & (Rv2) CCGATGATCTGTTCAGCCG \\
\hline & (Fw3) ATTTCTTCCTCGTGGCGGGC & (Rv3) TCGCCAGAAGCGTAATGCC \\
\hline
\end{tabular}

QRDR, quinolone resistance-determining region. 
Table 2

Selection of resistant mutant strains M1 to M8 from Brucella melitensis $16 \mathrm{M}$, with resulting minimum inhibitory concentrations (MICs) to moxifloxacin (MFX), ciprofloxacin (CIP) and levofloxacin (LVX), and characterised GyrA quinolone resistance-determining region (QRDR) substitutions

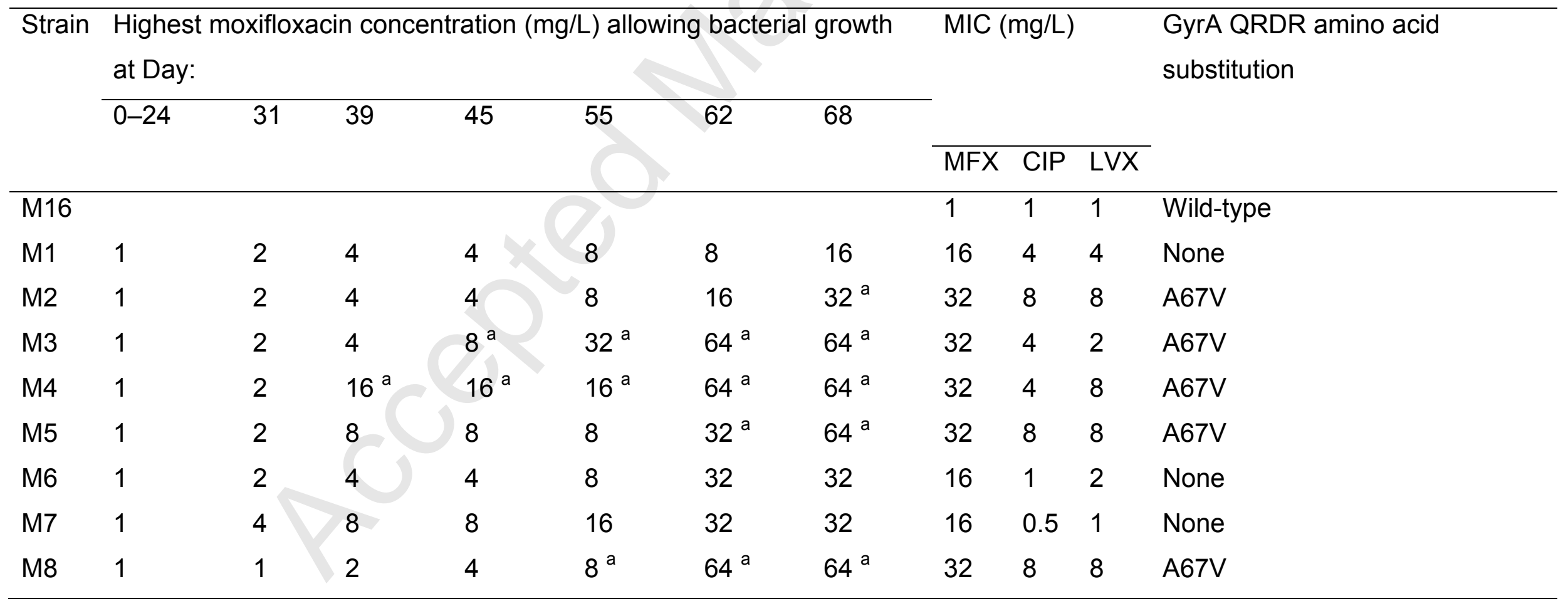




\begin{tabular}{|c|c|c|c|c|c|c|}
\hline Escherichia coli & Serine & 0.006 & Alanine & 0.032 & $\times 4$ & {$[27]$} \\
\hline Mycobacterium fortuitum & Serine & 0.06 & & & & {$[28]$} \\
\hline Mycobacterium tuberculosis & Alanine & 1 & Valine & 8 & $\times 8$ & [29] \\
\hline Brucella abortus & Alanine & 0.5 & Valine & $32 / 64$ & $\times 64-128$ & [25] \\
\hline B. melitensis & Alanine & 1 & Valine & $4-8$ & $\times 4-8$ & Present work \\
\hline
\end{tabular}

${ }^{\text {a }}$ Presence of GyrA A67V substitution.

${ }^{\mathrm{b}}$ Escherichia coli numbering system (position GyrA 67 in B. melitensis).

${ }^{\mathrm{c}}$ MIC to ofloxacin and/or ciprofloxacin. 
Table 3

Moxifloxacin minimum inhibitory concentrations (MICs) (mg/L) of Brucella melitensis 16M and the eight moxifloxacin-resistant mutants in the presence or absence of efflux pump inhibitors (EPIs)

\begin{tabular}{|c|c|c|c|c|c|c|c|c|c|c|c|c|c|c|}
\hline \multirow[t]{3}{*}{ Strain } & \multicolumn{8}{|c|}{ Moxifloxacin } & \multirow{2}{*}{\multicolumn{3}{|c|}{$\begin{array}{l}\text { Ciprofloxacin + PABN } \\
(\mu \mathrm{M})\end{array}$}} & \multirow{2}{*}{\multicolumn{3}{|c|}{$\begin{array}{l}\text { Levofloxacin + PA } \beta N \\
(\mu \mathrm{M})\end{array}$}} \\
\hline & \multirow{2}{*}{$\begin{array}{l}\text { No } \\
\text { EPI }\end{array}$} & \multirow{2}{*}{$\begin{array}{l}\text { Ver } 25 \\
\mu \mathrm{M}\end{array}$} & \multirow{2}{*}{$\begin{array}{l}\text { Res } 32.8 \\
\mu \mathrm{M}\end{array}$} & \multirow{2}{*}{$\begin{array}{l}\text { SO } 50 \\
\mu \mathrm{M}\end{array}$} & \multirow{2}{*}{$\begin{array}{l}\text { NMP } 88.4 \\
\mu \mathrm{M}\end{array}$} & \multicolumn{3}{|c|}{ PA $\beta N(\mu \mathrm{M})$} & & & & & & \\
\hline & & & & & & 38.5 & 77 & 154 & 38.5 & 77 & 154 & 38.5 & 77 & 154 \\
\hline $16 \mathrm{M}$ & 1 & 1 & 1 & 1 & 1 & 0.5 & 0.5 & 0.5 & 0.5 & 0.5 & 0.5 & 1 & 1 & 0.5 \\
\hline M1 & 16 & 16 & 8 & 8 & 8 & 8 & 4 & 2 & 2 & 2 & 2 & 2 & 2 & 2 \\
\hline M2 & 32 & 32 & 32 & 32 & 16 & 16 & 8 & 4 & 16 & 8 & 8 & 16 & 16 & 8 \\
\hline M3 & 32 & 32 & 32 & 32 & 16 & 16 & 8 & 4 & 16 & 8 & 8 & 16 & 16 & 8 \\
\hline M4 & 32 & 32 & 16 & 32 & 16 & 32 & 16 & 16 & 8 & 8 & 8 & 8 & 8 & 4 \\
\hline M5 & 32 & 32 & 16 & 32 & 16 & 16 & 8 & 4 & 16 & 8 & 8 & 8 & 8 & 4 \\
\hline M6 & 16 & 8 & 16 & 8 & 8 & 16 & 8 & 4 & 4 & 2 & 2 & 4 & 4 & 4 \\
\hline M7 & 16 & 16 & 16 & 16 & 8 & 8 & 4 & 2 & $<0.5$ & $<0.5$ & $<0.5$ & 1 & 1 & $<0.5$ \\
\hline M8 & 32 & 32 & 32 & 32 & 16 & 8 & 8 & 4 & 8 & 8 & 4 & 8 & 8 & 4 \\
\hline
\end{tabular}

Ver, verapamil; Res, reserpine; SO, sodium orthovanadate; NMP, 1-naphtylmethyl-piperazine; PA $\beta N$ : phenylalanine-arginine- $\beta$ naphthylamide. 